\title{
The review on Epidemiology, Prevention, Control and Surveillance of Nosocomial Infections
}

Dattatreya Mukherjee (Student Id: 2016059300)

MBBS Student 2016 Batch, Jinan University, P.R China

\section{$\underline{\text { Abstract: }}$}

Nosocomial infection or hospital associated infections occur to the patients who are admitted inside the hospital. This occurs both in developed and developing countries with a measure of $7 \%$ in developed and $10 \%$ in developing countries. According to $\mathrm{WHO}$, as this infection occurs after 48 hours of hospital admission so it prolongs the duration of the admission and it increases the economic burden. There is most common aetiology of nosocomial infections are catheter induced infection, surgical sight infections and ventilation associated infections. According to WHO upper respiratory tract infection is the most common nosocomial infections. Nosocomial pathogens are bacteria, Virus and funguses. Patient acquired this infection through hospital environments and people who are surrounded the patients, so in prevention, discontinuation of the transmission chain is very important. Hospital waste is a possible source of contaminants and 20-25\% of the sources are dangerous. Nosocomial infections can be managed by a policy for disease prevention, the use of antibiotics and surveillance about antibiotic resistance and the implementation of antibiotic management policies. A good protocol and surveillance system can reduce the Nosocomial infections. This is a detail review of 5 years on epidemiology, prevention, control and surveillance of Nosocomial infections or Hospital associated infections

Key Words: Nosocomial Infections, Catheter associated infections, Post-surgical infections, Antibiotic resistaOnce, prevention, treatment, surveillance

Total Word (With Reference): 5513

Total Reference: 35

Total Page: 28

Total Fig: 9, Total Table: 9 


\section{Introductions:}

Nosocomial infections are also called hospital associated infections and it can be defined as,

An infection acquired in hospital by a patient who was admitted for a reason other than that infection (1). An infection occurring in a patient in a hospital or other health care facility in whom the infection was not present or incubating at the time of admission. This includes infections acquired in the hospital but appearing after discharge, and also occupational infections among staff of the facility (2).

According to WHO, infections occurring after 48 hours of hospital admission is granted as a nosocomial infection. There are couple of infection sites like urinary tracts or blood vessel infections. According to WHO more than 50 infection sites are identified. These are achieved by different data published by $C D C(3,4)$, different international conferences and difference surveillance reports and more than 50 potential sites of infections are identified.(5)

In various time medical staffs got this infection as an occupational hazard (6) and invasive devices are key etiology of the nosocomial infections in the hospital admitted patients. (7)

In every hundred hospitalized patients, seven patients in developed countries and 10 patients in developing countries have nosocomial infections. (8)

Nosocomial infections can be endemic or epidemic. Endemicity is more common but epidemic also seen when a pandemicity arrived to that area or country.

ICU, burn units, pediatric unit, geriatric unit and HIV units patients are the most susceptible to the nosocomial infections as they are immune compromised. According to EPIC II study the incidence of nosocomial infection in ICU patients are as high as 51\%. (9) In an extensive study it has been seen that in USA and UK nosocomial infection density is 13.0 and 20.3episodes per thousand patientdays.(10) 
Candida auris is called super bug and a night mare for the hospital management. One patient in Israel got C. auris. The patient had a travel history from south Africa, later it was proved that it came from $S$ Africa, but unfortunately another patient in that hospital affected to candida and it's an example of transmission chain of nosocomial infections. (11)

\begin{tabular}{|l|l|}
\hline $\begin{array}{l}\text { Type of } \\
\text { Nosocomial Inf. }\end{array}$ & Simplified Criteria \\
\hline Surgical Infections & $\begin{array}{l}\text { Any purulent discharge, abscess, or spreading cellulitis at } \\
\text { the surgical site during the month after the operation }\end{array}$ \\
\hline Urinary Infection & $\begin{array}{l}\text { Positive urine culture (1 or } 2 \text { species) with at least 105 } \\
\text { bacteria/ml, with or without clinical symptoms }\end{array}$ \\
\hline $\begin{array}{l}\text { Respiratory } \\
\text { Infections }\end{array}$ & $\begin{array}{l}\text { Respiratory symptoms with at least two of the following } \\
\text { signs appearing during hospitalization: } \\
\text { - cough } \\
\text { - purulent sputum } \\
\text {-new infiltrate on chest radiograph consistent with } \\
\text { infection }\end{array}$ \\
\hline $\begin{array}{l}\text { Vascular Catheter } \\
\text { infection }\end{array}$ & $\begin{array}{l}\text { Inflammation, lymphangitis or purulent discharge at the } \\
\text { insertion site of the catheter }\end{array}$ \\
\hline Septicemia & \begin{tabular}{l} 
Fever or rigours and at least one positive blood culture \\
\hline
\end{tabular}
\end{tabular}

Table 1: Simplified criteria of surveillance of nosocomial infections (WHO/CDS/CSR/EPH/2002.12)

The prevention is possible by stopping the transmission chain. Nosocomial infections also increase the stay in the hospital and increase the economic burden and mortality rate. (12)

In this review we have discussed in details about epidemiology, prevention, control and surveillance of nosocomial infections

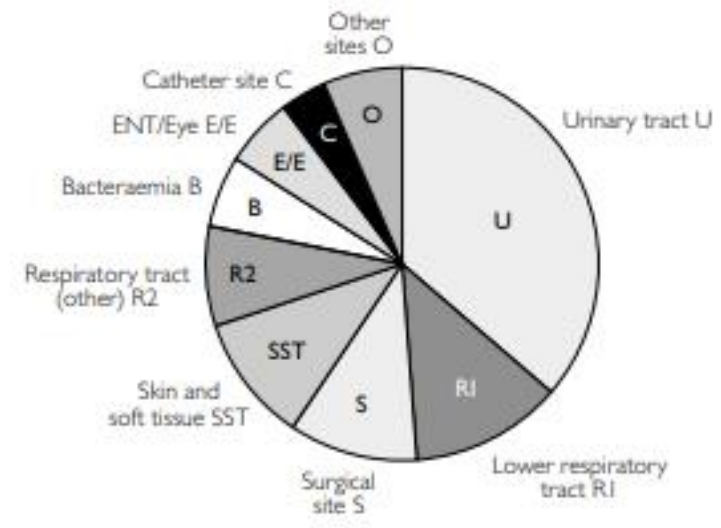


Fig1. Rate of incidence w.r.t possible infection sites

\section{Types of Nosocomial infections:}

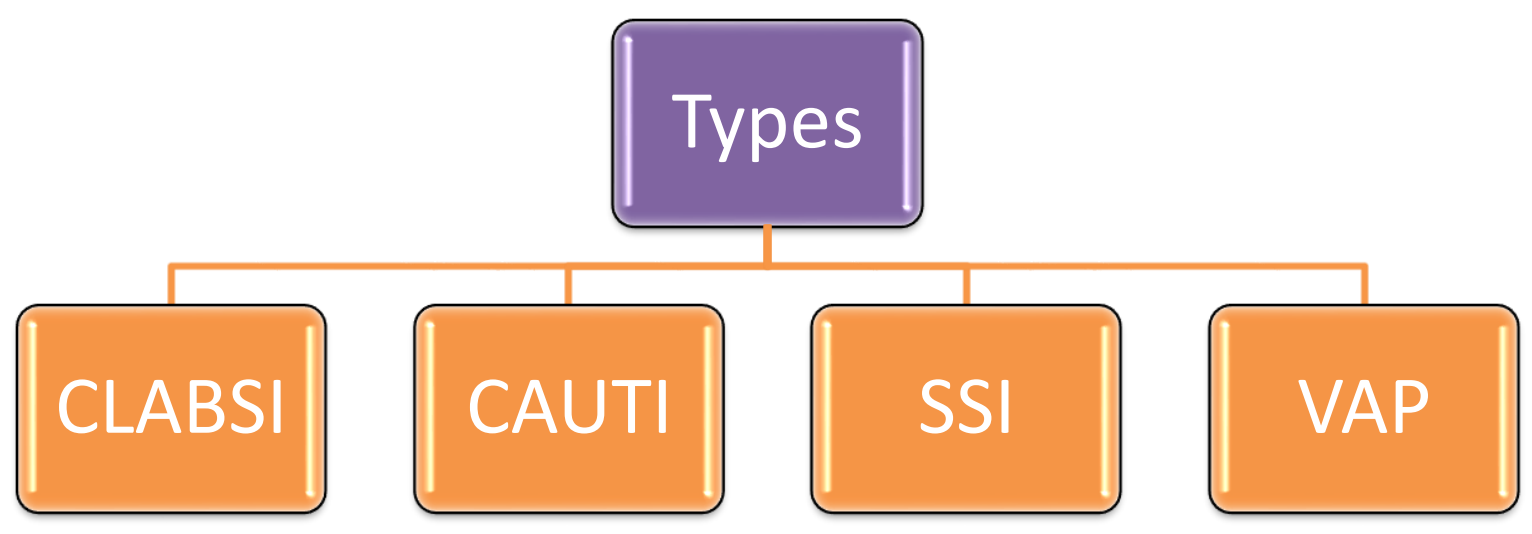

Table 2: Types of nosocomial infections

CLABSI: Central line associated blood stream

CAUTI: Catheter associated urinary tract infections

SSI: Surgical site infections

VAP: Ventilator associated pneumonia

a. Central Line Associated Blood Stream (CLABSI)

CLABSI is a common infection which occurs from central lines. Incidence rate is 12-25\%. (13) Although in developed countries the amount of CLABSI is decreasing due to proper prevention measures. $(14,15)$

b. Catheter Associated Urinary Tract Infections (CAUTI)

CAUTI is the most common global nosocomial infections. More than $12 \%$ is urinary tract infections. Endogenous bacteria are formed and formed UTI and cystitis, orchitis, epididymis and prostatitis. (16) 
c. Surgical Site Infections: (SSI)

2-5\% patients are subjected to surgical site infections. Proper dressings and sterilization techniques are important to stop this. Some places these guidelines are not followed so that SSI are as high as 20\%. The main cause of SSI is endogenous microflora like Staphylococcus aureus. $(17,18)$

d. Ventilator Associated Pneumonia (VAP)

9-27\% patients suffered from VAP.(19) It occurs within 48 hours of endotracheal intrubation. 86\% pneumonia is associated to VAP.(20) Fever, leukopenia are common.

Other Nosocomial infections:

1. Skin and Soft tissue infections

2. Gastrointestinal nosocomial infections

3. Sinusitis and ocular infections

4. Endometriosis and other gynaecological infections during child birth

\section{Nosocomial Pathogens:}

Nosocomial infections are caused by different pathogens like bacteria, virus, fungus etc. Fungi is the most common causative agent and a big challenge in antibiotic resistance. Like Pseudomonas, antibiotic resistance is a big challenge. So many genetic engineering and molecular techniques are applied to reduce the antibiotic resistance.

Bacteria (Table 3)

Commensal Bacteria

It is normally present in everyone's body. When the subject become immune compromised then they affected and cause infection by invading the blood vessels. 


\begin{tabular}{|l|l|}
\hline & $\begin{array}{l}\text { Example: } \text { Cutaneous Coagulase } \\
\text { negative Staphylococcus } \\
\text { E coli which is present in intestine }\end{array}$ \\
\hline Pathogenic Bacteria & Gram negative: Clostidium, Ecoli, \\
& proteus, Kleibsiella, enterobactor, \\
Pseudomonas, & Gram positive: S. aureus \\
& Legionella \\
\hline
\end{tabular}

Viruses: ( Table:4)

\begin{tabular}{|l|l|}
\hline Viruses & Hepatitis B and C \\
& Respiratory syncitial virus \\
& Enterovirus \\
& Cytomegalo virus \\
& Ebola \\
& Influenza \\
& HSV \\
& Varicella zoster \\
\hline
\end{tabular}

Parasites and Fungi (Table: 5)

\begin{tabular}{|l|l|}
\hline Parasites and fungi & $\begin{array}{l}\text { Giardia, Candida, Aspergillus, } \\
\text { Cryptococcus, Cryptosporidium, } \\
\text { Sarcoptes }\end{array}$ \\
\hline
\end{tabular}




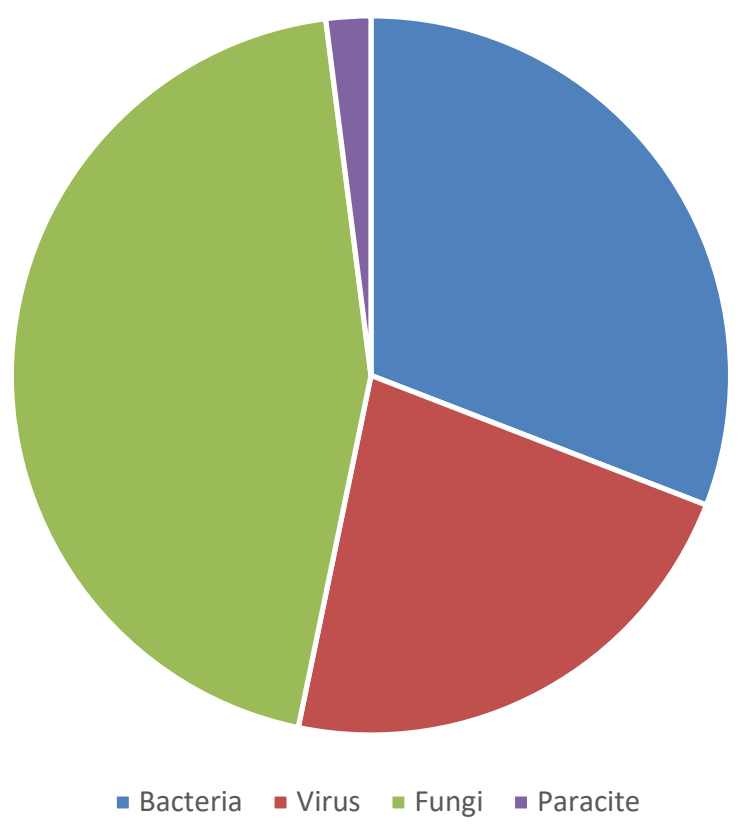

Fig 2 rate of causative agent w.r.t nosocomial infection agent

- Most common Bacteria induced nosocomial infection is Staphylococcus infection(18.5\%) followed by E coli (16.5\%) (21)

- Most common fungal induced nosocomial infection is Candida sp.

\section{Transmission of infection:}

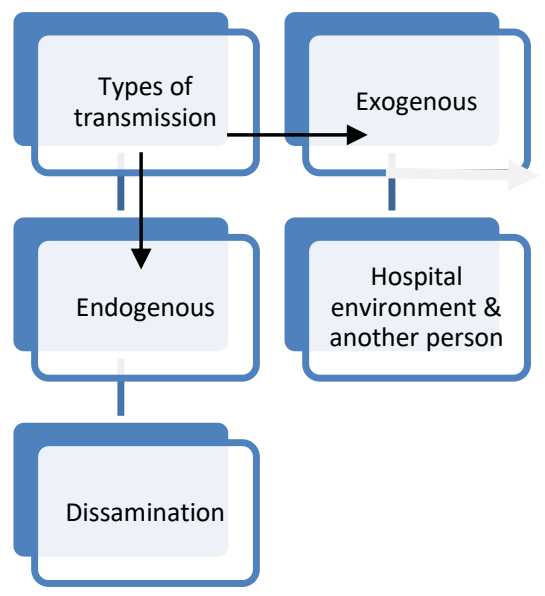

Table 6: Types of transmission chain (WHO) 
Endogenous transmission is the transmission is the transmission which occurs due to dissemination of commensal microbes. Like E coli is present in intestine and disseminated to urinary tract causing UTI.

Exogenous infection can be happened from hospital environment and the persons like hospital staffs, visitors etc.

\section{Routes of Transmission:}

a. Contact Transmission: Direct Transmission and Indirect Transmission

Direct Transmission: Direct dissemination occurs with the movement of infectious agents from One person to another, e.g. the blood of a patient entering the healthcare worker's (HCW's) body from a cut in the skin that is unprotected.

Indirect Transmission: The movement of an infectious agent into an infectious agent requires indirect transmission.

Contaminated intermediate entity or person, e.g. transmitting hands of an $\mathrm{HCW}$ after touching a contaminated body site, infectious agents on one patient without before contacting another human, performing hand hygiene, or an HCW coming action with fomites or faeces (e.g. bedding) and then with a customer.

b. Droplet Transmission: When an infected human coughs, sneezes or speaks, droplet transmission occurs. Infectious particles with $>5$ microns in size are droplets. The distribution range of droplets is restricted by the force of expulsion and gravity and droplets may also be transferred indirectly to the mucosal surfaces, typically 1 meter. Examples of infectious agents that are passed down by droplets, The influenza virus, Bordetella pertussis, and meningococcus are also included.

c. Airborne Transmission: Airborne transmission can occur via infectious agent-containing particles that over time and space, they remain suspended in the air. Aerosols of small particles ( $<5$ microns) they are created by breathing, speaking, coughing or sneezing, and secondarily by evaporation of larger droplets under low humidity conditions.

d. Vector Borne Transmission: Vector-borne transmission refers to the movement of vectors of microorganisms such as mosquitoes and can be 
avoided by suitable construction and maintenance an $\mathrm{HCF}$, with windows locked or screened, and good housekeeping. Malaria, dengue and chikungunya are examples of vector-borne illnesses.

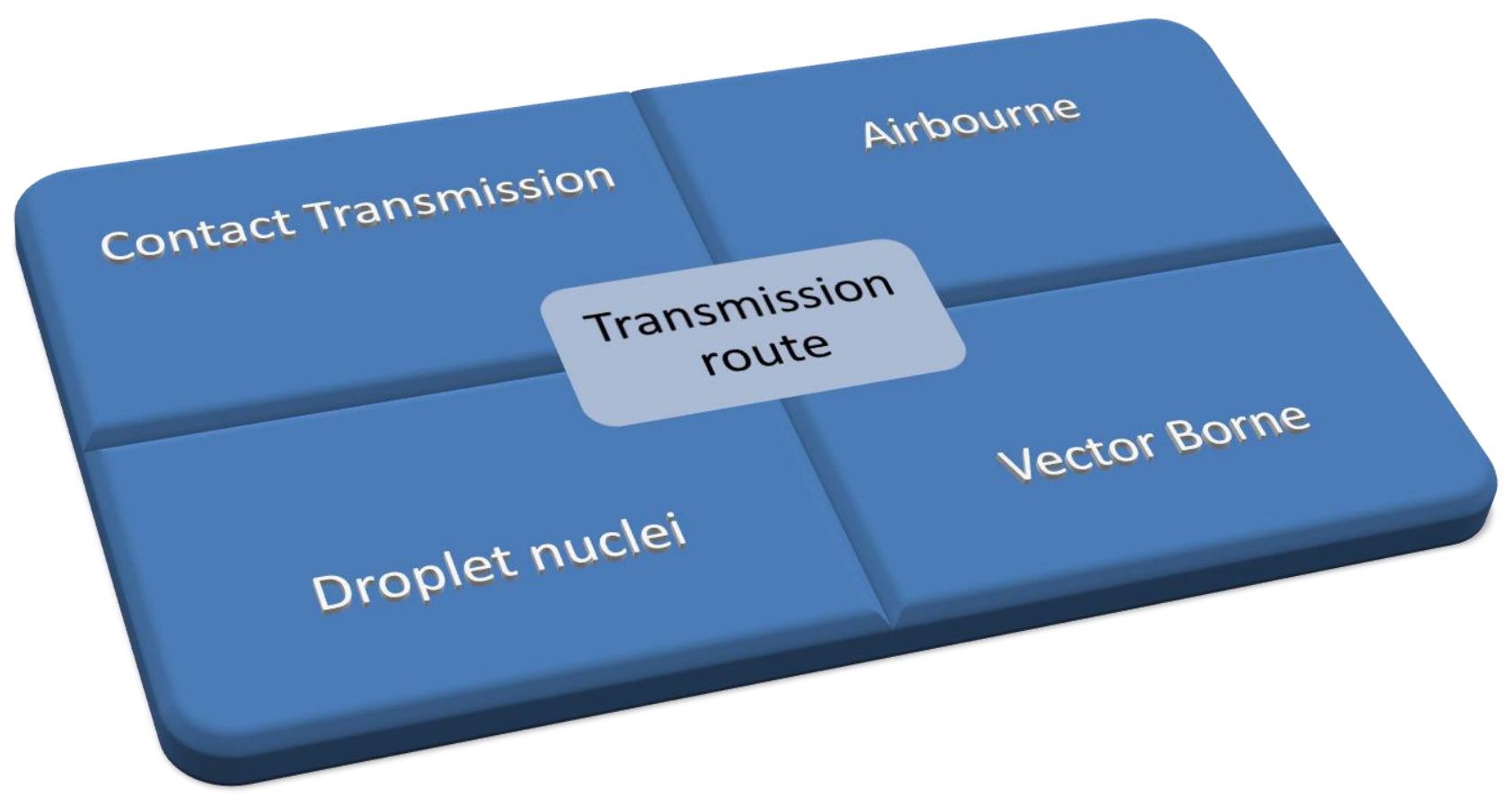

Fig 3: Transmission route (22)

So to prevent a nosocomial infection, break down of transmission chain is important. It is done by maintaining sterilization, hygiene and other strict guidelines.

\section{Determinants:}

Risk factors of nosocomial infections depends on many factors including environments, susceptibility and lack of awareness. 

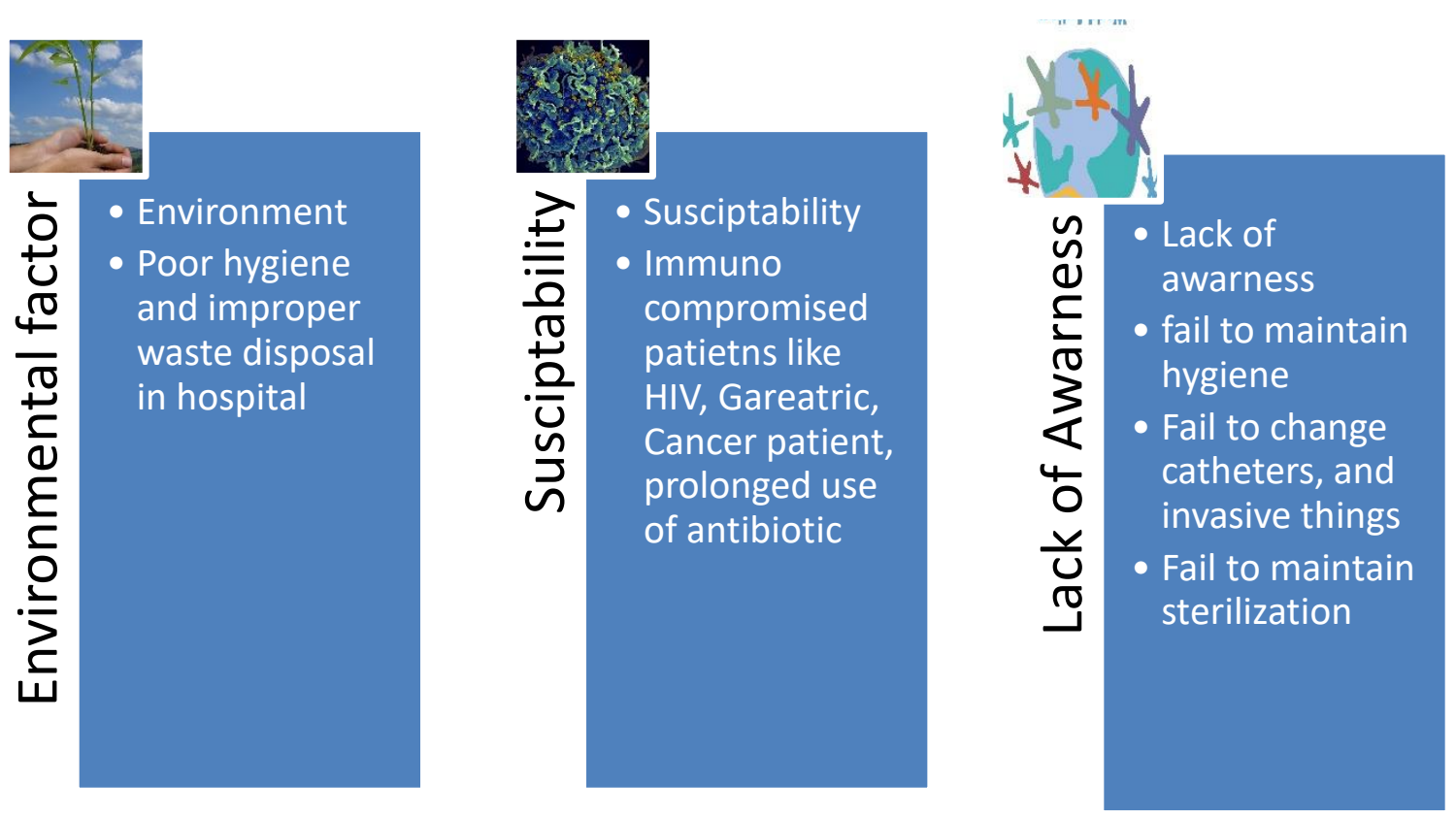

Table 7: Maintain proper hygiene, sterilization measures, proper waste disposal and continuous awareness program can reduce the amount of incidence of nosocomial infections.

\section{Control of Nosocomial infection:}

Now days many strict guidelines are followed, though there is a decrease in the rate of incidence, but still now incidence rate is quite high. In one day, one patient out of 25 patients affects to nosocomial infection.(23).

\section{a. Infection Control Programmes:}

Various hospitals and organization provides trainings on infection control. Infection control in different sector is very important. Hospital staffs and visitors should be trained and a supervision on hygiene and sterilization measures should be followed. Infection Control Committee should be made to improvise the new rules and guidelines. (24) 


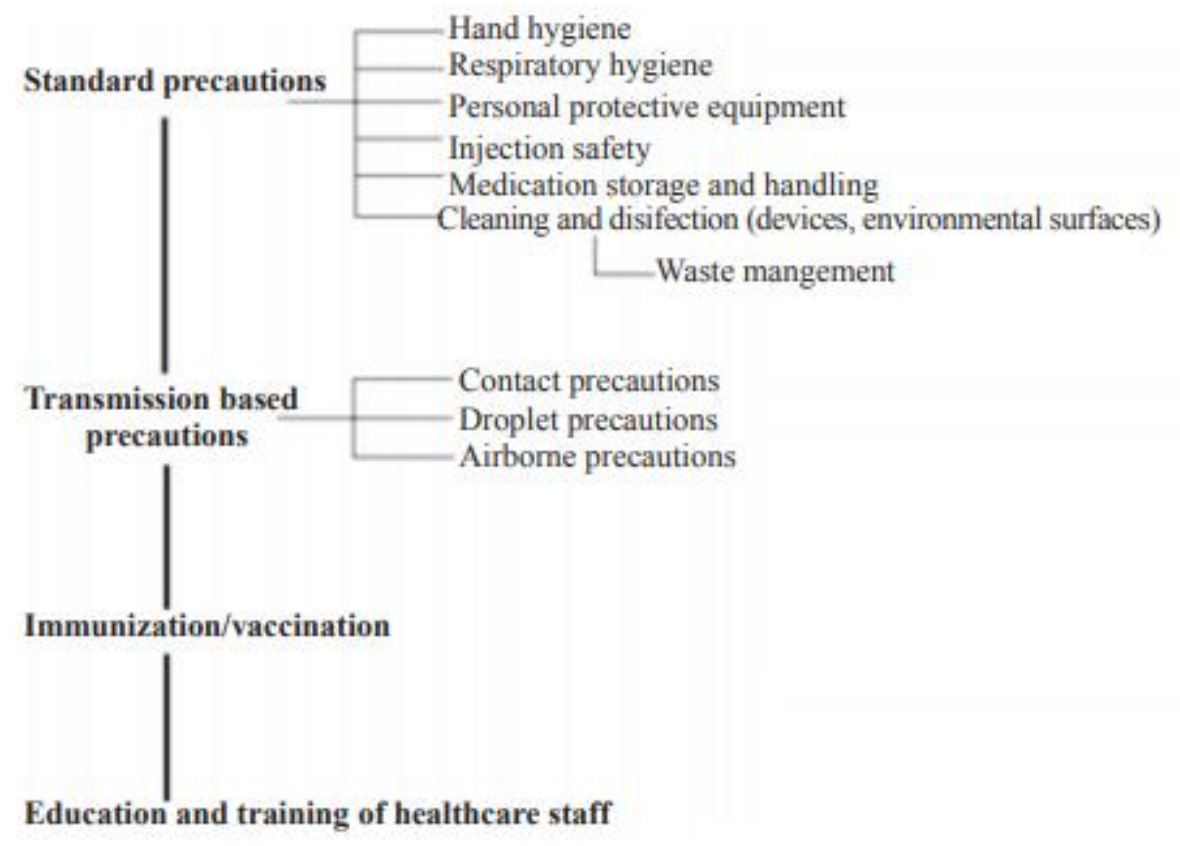

Fig 4: Nosocomial infection Control (25)

\section{Basics Concepts on prevention of Nosocomial Infections: (2020)(22)}

HAIs can be prevented by breaking the epidemiological triad. The most effective way to prevent HAI is by introducing a barrier between the susceptible host and the infecting organism. Most HAIs can be prevented through readily available and relatively inexpensive strategies such as compliance with recommended infection prevention practices such as:

$x$ Hand hygiene

$x$ Appropriate use of personal protective equipment (PPE)

$x$ Following aseptic techniques stringently

$x$ Paying attention to established practices for cleaning and decontamination of soiled instruments, followed by either sterilization or high-level disinfection $x$ Appropriate disposal of biomedical waste (BMW)

$x$ Appropriate cleaning and disinfection of the environment 
$x$ Improving safety in operating rooms and other high-risk areas where the most vulnerable patients are housed and there is a high risk of exposure to infectious agents

$x$ Maintaining a safe working environment and safe work practice

Treatment programmes contain a selection of evidence-based interventions to be introduced together a major increase in health treatment may be shown they have collectively together a stronger influence on the result than the independent application of particular steps

Bundle adherence aims to provide a stable and secure patient with Care. Sets of bundles for the prevention of central line-associated bloodstream infections (CLABSIs), bundles for the prevention of catheter-associated urinary tract infections (CAUTIS), bundles for the prevention of VAP, and bundles for the prevention of SSIs are treatment bundles that have shown a substantial effect on HAI prevention.

\section{Prevention of Nosocomial infection in different part of the hospital:}

Surgical Unit, ICU, Ventilation Associated Pneumonia, Catheter induced pneumonia

\section{Prevention in Surgical Unit:}

Personnel Hand/forearm antisepsis for surgical team members is of crucial importance. Nails should be kept short and all jewellery, artificial nails or nail polish should be removed before surgical hand preparation.

1. Hands should be washed and debris should be removed from underneath fingernails using a nail cleaner (not brushes), preferably under running water. Sinks should be designed to reduce the risk of splashes.

2. Surgical hand antisepsis should be performed using either a suitable antimicrobial soap, before donning sterile gloves.

3. A preoperative surgical hand scrub should be done for at least 5 minutes using an appropriate antiseptic scrub. Hands and forearms should be cleaned up to the elbows. 
4. After performing the surgical hand scrub, hands should be kept up and away from the body (elbows in flexed position) so that water runs from the tips of the fingers toward the elbows and not vice versa.

5. If running water is not available, clean stored water can be used. Water should be stored in a bucket with a tap at one side to dispense water. If such a bucket is not available, clean water can be poured on the hands with the help of a container with a long handle. Another person should pour the water.

6. If the quality of water is not assured in the OT, surgical hand antisepsis using ABHR is recommended. A sufficient amount of ABHR should be applied to dry hands and forearms for the length of time recommended by the manufacturer, typically 1.5 minutes, and hands and forearms allowed to dry before donning sterile gloves.

RUB HANDS FOR HAND HYGIENE! WASH HANDS WHEN VISIBLY SOILED

(1) Duration of the entire procedure: $20-30$ seconds

1 a

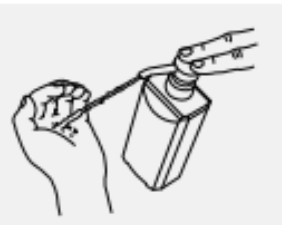

Apply a palmful of the product in a cupped hand, covering all surfaces;

3

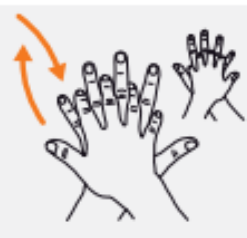

Right paim over left dorsum with interlaced fingers and vice versa;

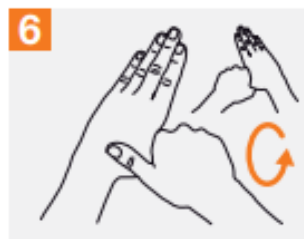

Rotational rubbing of left thumb clasped in right palm and vice versa;
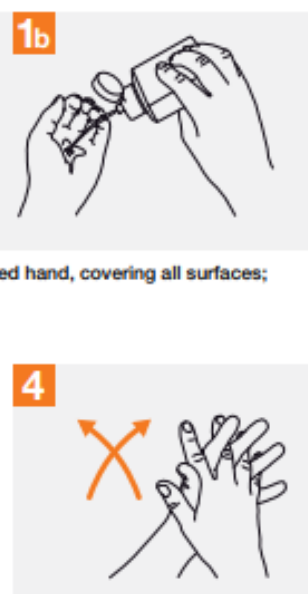

Palm to palm with fingers interlaced;

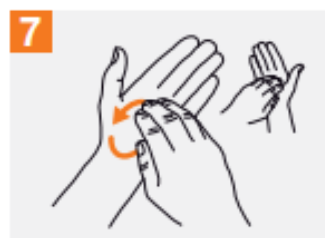

Rotational rubbing, backwards and forwards with clasped fingers of right hand in left palm and vice versa;

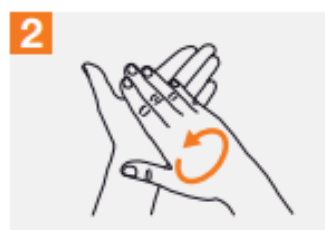

Rub hands paim to paim;

5

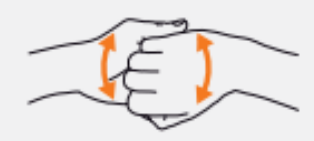

Backs of fingers to opposing paims with fingers interlocked;

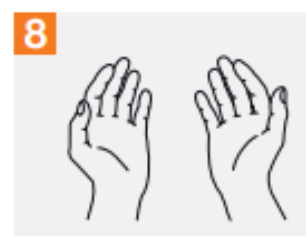

Once dry, your hands are safe.

Fig 5: Hand Washing and Scrub (WHO) 
Scrubs Microorganisms are constantly shed from the hair and skin of persons and also from their clothes. Microorganisms are also expelled through respiratory secretions while breathing, talking, coughing and laughing. "Scrubs" refers to the sanitary clothing worn by the OT staff, usually comprising a short-sleeve, v-neck shirt and loose-fitting, drawstring pants. The design of scrubs minimizes places where contaminants can hide, and they are easy to launder. They should be changed after a likely contamination and should always be cleaned in a healthcare laundering facility.

The surgical attire includes gloves, gowns, caps, mask, eye protection, waterproof aprons and footwear. It protects the patient from risk of infection from the hair, skin, clothes and respiratory secretions of the surgical team. The surgical attire also protects the surgical team from risk of exposure to blood and tissues of the patient during operation.

The sterile field: It is important to maintain a sterile field to prevent contamination of surgical incision. A sterile field is the area prepared around the surgical procedure site and where the sterile instruments and other items needed during the operation are placed. It is created by placing sterile towels or sterile drapes on the prepared procedure site on the patient and includes a stand nearby. Only sterile objects and persons in surgical attire (scrubbed team) are allowed within this field. Areas above the chest and below the waist of the scrubbed team are considered non-sterile. Items outside and below the draped area are considered non-sterile. The field is considered non-sterile if a non-sterile object or nonscrubbed person comes within the sterile field.

A clean operating environment is essential to prevent SSI. The OT is cleaned and disinfected to prevent microbial contamination. Exogenous sources of infection in the OT are: people, anaesthesia equipment, surfaces such as walls floors and there should be no dust in the OT; dust settling on the sterile fi eld can carry microorganisms particularly in operations of long duration. Dust may be acquired from the outside environment due to defective filtration of air. Lint-containing textiles can be a source of dust as also floor mops. Dust particles can be reduced by good laundry practices to reduce the formation of lint and by the use of wet vacuum on the floor. 


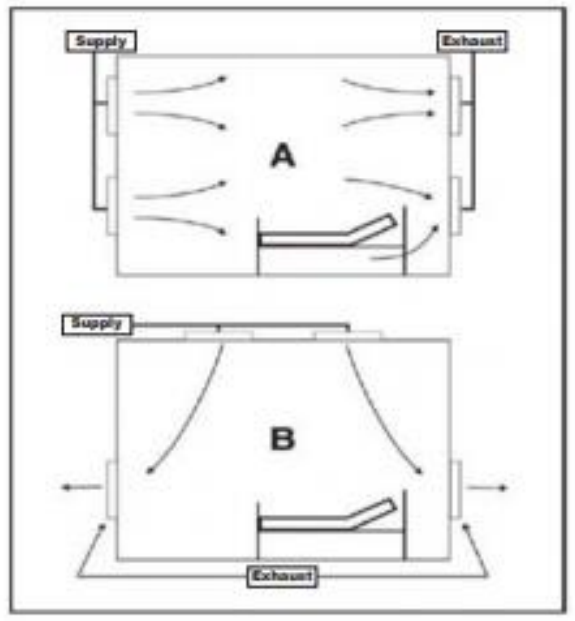

Fig 6.1: Ventilation (Indian Guidelines)
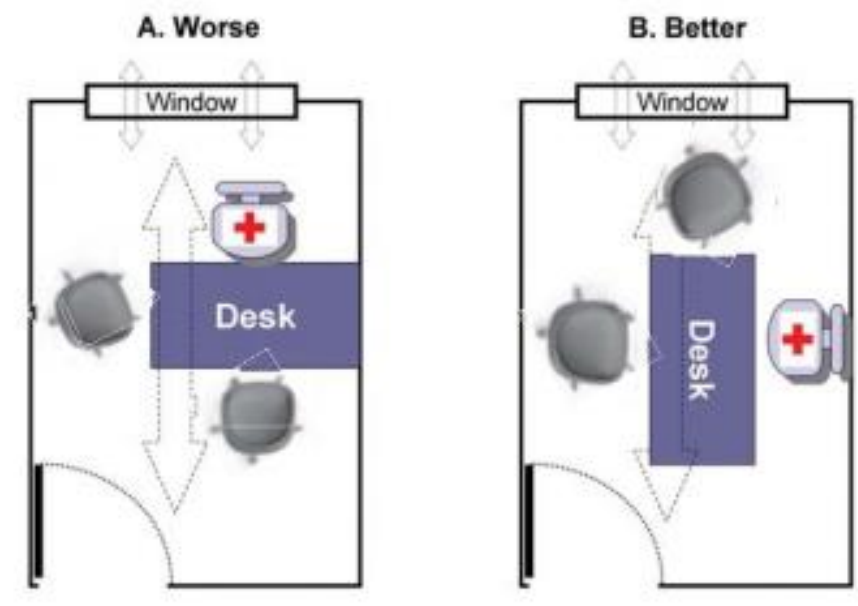

Fig 6.2: Ventilation (Indian Guidelines)

Surgical antimicrobial prophylaxis: Surgical antimicrobial prophylaxis (SAP) should be administered before the surgical incision when indicated (depending on the type of operation). This should be based on the hospital antibiotic policy. The initial dose of prophylactic antimicrobial agent should be administered by the intravenous route, timed such that a bactericidal concentration of the drug is established in serum and tissues when the incision is made. Administration of SAP should be within 120 minutes of incision, while considering the half-life of the antibiotic. Clinicians should consider the half-life and protein binding as the most important pharmacokinetic parameters of any single SAP agent in order to ensure 
adequate serum and tissue concentration at the time of incision and during the entire surgical procedure. SAP should not be prolonged after the completion of the operation and is not recommended in the presence of a wound drain for the purpose of preventing SSI. To reduce the stay in hospital, patients are discharged before incision has healed. The patient should be educated as to how to take care of the incision site, personal hygiene, about signs and symptoms of infection and whom to contact if infection occurs.

\section{Prevention on ICU induced HAI:}

Intensive care units (ICUs) house patients that are particularly vulnerable and at five- to ten-times at higher risk of HAI. With defences compromised due to various invasive devices such as peripheral and central lines, urinary catheters and mechanical ventilators, they are particularly prone to device-related infections. Intrinsic factors such as immunosuppression and comorbidities compound their vulnerabilities. Patients in the ICU are also exposed to broad-spectrum antibiotics and are susceptible to multidrug-resistant organisms such as Acinetobacter spp. and Pseudomonas spp. (25)

a. In high-income countries, approximately $30 \%$ of patients in ICU are affected by at least one HAI.

b. In LMICs the frequency of ICU-acquired infection is at least 2-3 fold higher than that in high-income countries; device-associated infection densities are up to 13 times higher than those in the USA.

c. Similarly, newborns admitted in NICUs are at higher risk of acquiring HAI in developing countries, with infection rates 3 to 20 times higher than those in high-income countries.

Patients with risk factors:

1. Age $>70$ years

2. Shock, major trauma, acute renal failure, coma

3. Prior antibiotics $x$

4. Mechanical ventilation $x$ Indwelling catheters

5. Immunocomromised patients on steroids or chemotherapy

6. Prolonged ICU stay (>3 days) 
Main preparation is maintaining dress and skin hygiene and use ICU footware.

\section{Prevention of Ventilation Associated Pneumonia:}

Pneumonia is the second most common HAI reported in the world and is associated with substantial morbidity and mortality. Most patients with health care associated pneumonia are those with extremes of age, severe underlying disease, immunosuppression, depressed sensorium and cardiopulmonary disease, and those who have had thoraco-abdominal surgery. Most bacterial health care associated pneumonia occur by aspiration of bacteria colonizing the oropharynx or upper gastrointestinal tract of the patient. Intubation and mechanical ventilation greatly increase the risk of bacterial pneumonia because they alter first-line patient defences. Pneumonia due to infective causes occurring in a patient on mechanical ventilation is termed ventilator-associated pneumonia or VAP.

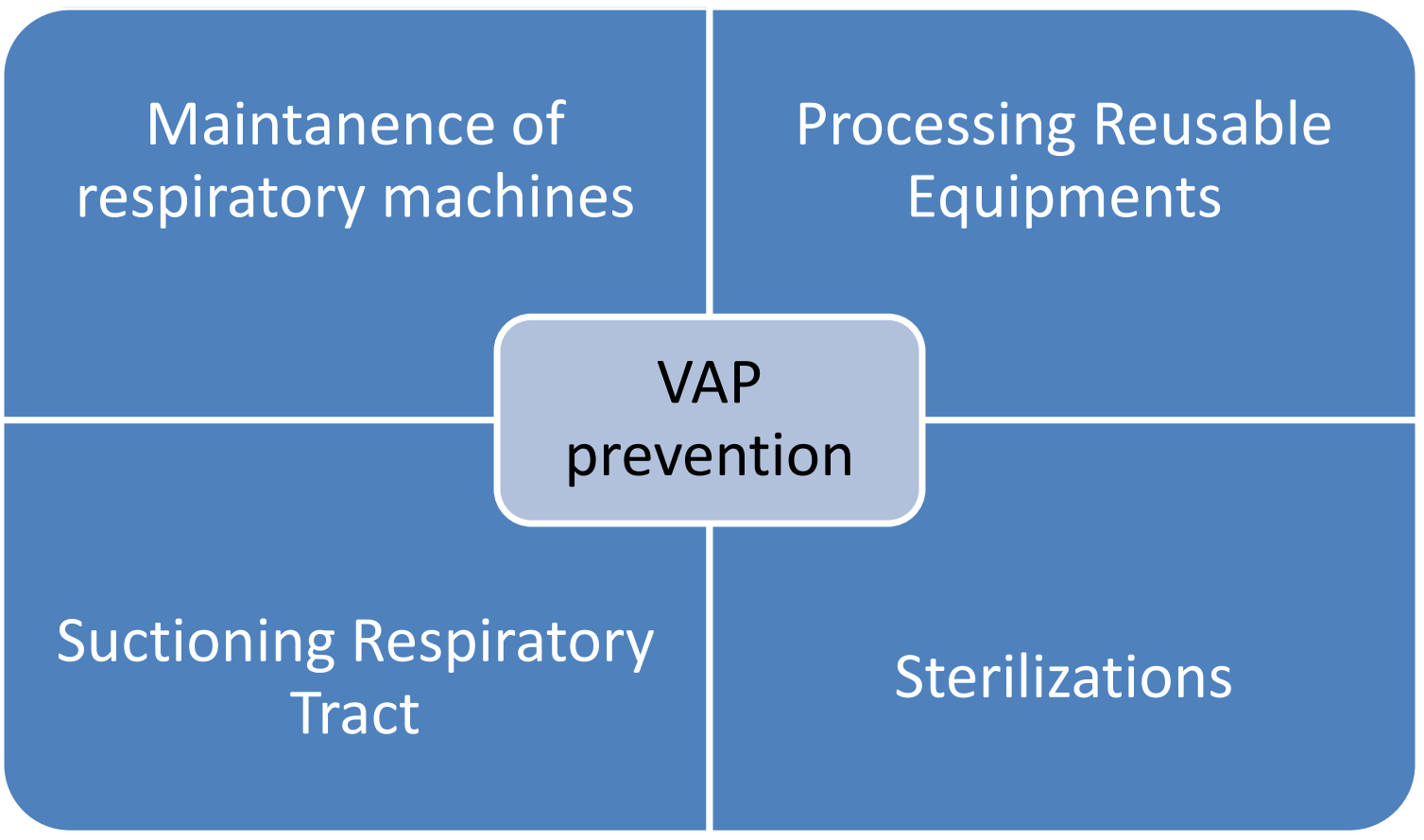

Fig 7 : Prevention of VAP (26)

Prevention of Catheter associated infections:

a. Education and training to the staffs

b. Selecting the best insertion site 
c. Asceptic technique during catheter

d. Skin Preparation and asceptic teq: Prepare clean skin with an antiseptic (70\% alcohol, tincture of iodine, an iodophor or chlorhexidine gluconate) before insertion of a peripheral venous catheter. Prepare clean skin with a $>0.5 \%$ chlorhexidine preparation with alcohol before insertion of a central venous catheter or peripheral arterial catheter and during dressing changes. If there is a contraindication to chlorhexidine, use tincture of iodine, an iodophor or $70 \%$ alcohol. Antiseptics should be allowed to dry according to the manufacturer's recommendation before placing the catheter

e. Prophylactic teq : Systemic antimicrobials should not be routinely administered before insertion or during use of a central venous catheter to prevent catheter colonization..

Exchange of the catheter regularly is very important to reduce the Catheter induced nosocomial infections.

\begin{tabular}{|l|l|}
\hline Clean gloves & $\begin{array}{l}\text { Peripheral intravascular catheter (if access site not touched } \\
\text { after application of skin antisepsis) }\end{array}$ \\
\hline Sterile gloves & $\begin{array}{l}\text { Arterial catheter } \\
\text { Central catheter } \\
\text { Midline catheter }\end{array}$ \\
\hline Sterile gloves & Guide-wire exchange \\
\hline Clean or sterile gloves & Changing dressing on intravascular catheter \\
\hline
\end{tabular}

Table 8: Different types gloves used in different situations

\section{Catheter induced Urinary Tract Infections and prevention:}

\section{Symptomatic UTI}

Prolonged catheterization

Female gender

Older age

Impaired immunity

\section{Bacteriuria}

Disconnection of drainage system Lower professional training of inserter Placement of catheter outside of OT Incontinence

Diabetes

Meatal colonization

Renal dysfunction

Orthopaedic/neurology services

Table 9: Risk Factors of UTI and Bacteriuria 
Urinary catheters shall be inserted only when necessary and left in place for as long as necessary. They should not be used solely for the convenience of patient-care personnel. Avoid use of urinary catheters for the management of urinary incontinence. Use urinary catheters in operative patients only when necessary rather than routinely. For operative patients who have an indication for an indwelling catheter, remove the catheter as soon as possible postoperatively, preferably within 24 hours, unless there are appropriate indications for continued use.

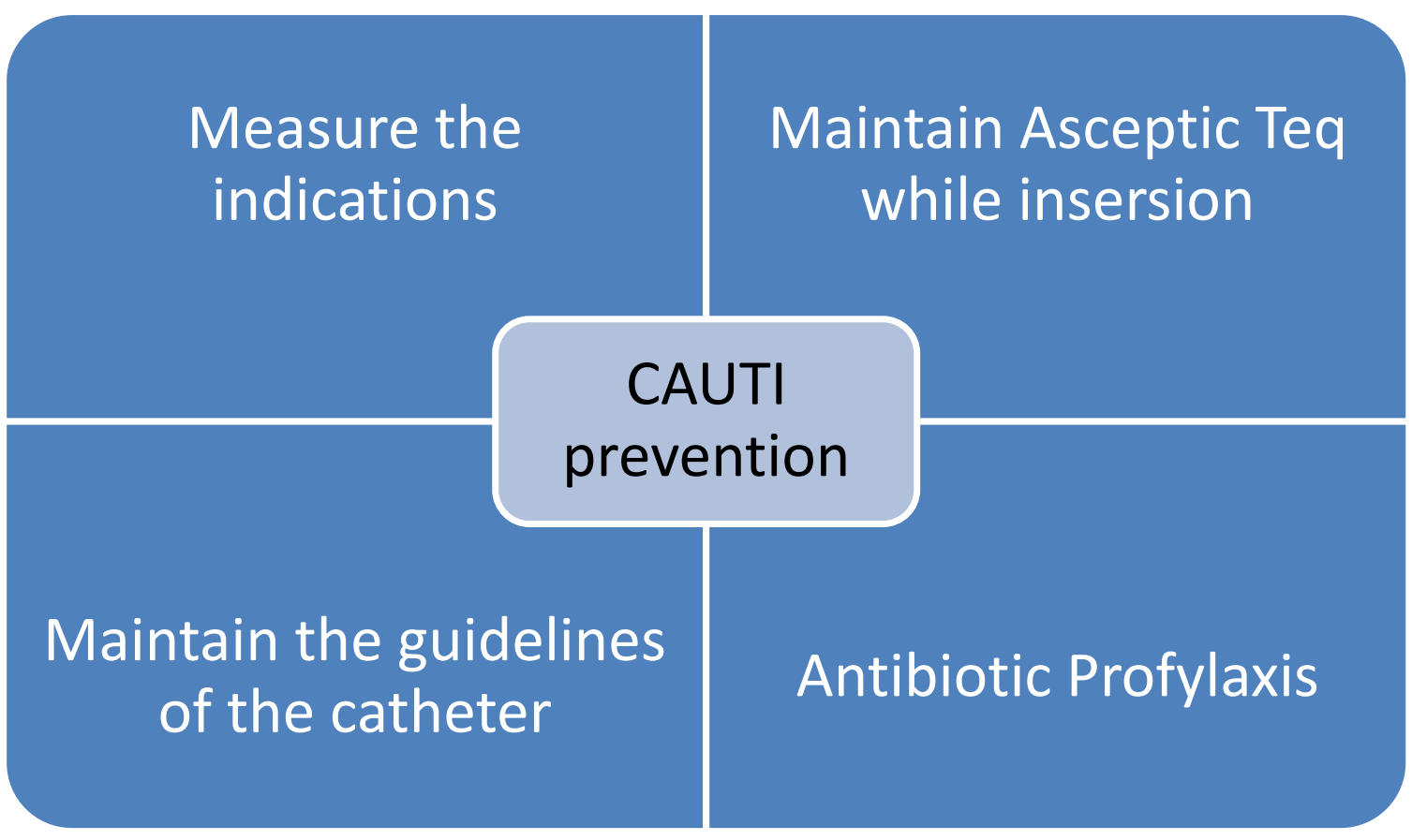

Fig 8 Prevention of CAUTI

Insert urinary catheter using aseptic technique

1. Hand hygiene

2. Catheter insertion kit with sterile gloves, drape, cleaning supplies

3. Sterile lubricant

4. Sterile urinary catheter attached to a drainage bag 


\section{Hospital Waste Management:}

Hospital waste may serve as a possible repository for contaminants that need careful treatment. 10-25 percent of the produced waste healthcare services are listed as unsafe. Healthcare Contagious waste should be processed in a restricted-access area. The Excess producing elevated heavy metal content and surgical waste, individuals contaminated with blood and sputum infection and the analytical labs must be individually disposed of. (28)

\section{Antimicrobial Resistance:}

Microbes are too small to see the creatures with the eyes, but anywhere on earth they are located. Antimicrobial Medicine drugs are used against live species that are pathogenic to bacteria. Resistance to antimicrobials occurs as the microbes develop the capacity to combat the drug effects; they are not killed and there is no stopping their development.

Antibiotics are commonly used to treat diseases. Usage of antimicrobials A proper health diagnosis of infection should be justified. Microorganism-causing the Centres for the Prevention of Diseases and Prevention (CDC) figures that about 100 million per annum Office-based doctors administer antibiotic courses, although roughly 50\% of these are unnecessary[29]. The antimicrobial collection should be based on the patient's in comparison to the essence of illness and bacteria, immunity.

Resistance to antibiotics is responsible for the death of an infant. In the SouthEast Asia zone, every five minutes. Drugs that have been used for the treatment of deadly diseases are now losing their effect because of emerging microorganisms that are immune to drugs (30) Self-medicine with antibiotics, improper dosage, extended use, lack of antibiotics standards for veterinary staff and animal misuse the key factors responsible for the rise in husbandry are about opposition. The successful management of this rebellion threatens UTI, pneumonia and bloodstream-causing infections with pathogens. Bacteria that are highly immune, such as MRSA or the cause of multidrug-resistant Gramnegative bacteria large worldwide prevalence rates of nosocomial infections. (31) South-East Asian region reports reveal that there a high resistance in E. 
coli and K. pneumoniae for third generation cephalosporin and more than quarter of S. aureus infections are methicillin resistant. (32) "Immediate action is needed to stop the world from heading towards pre-antibiotic era in which all achievements made in prevention and control of communicable diseases will be reversed", said Dr Poonam Khetrapal Singh, Regional Director of WHO South-East Asia Region (33)

\section{Control of Antibiotic Resistance:}

The current antibiotic resistance pandemic shows that it is motivated by antibiotics being overused and misused, which is a damage to the prevention and control of diseases. World report by the WHO on resistance to antibiotics, stopping the infection from occurring by an order to minimise the need for proper sanitation, clean water and vaccines, about antibiotics. The development of new diagnostics and other in healthcare institutions, instruments are required to keep ahead of the evolution of about opposition. Pharmacists should play their role of prescribing the right antibiotic when truly needed and policymakers should foster cooperation and information among all stakeholders (32)

\section{Surveillance of Nosocomial Infections:}

Although the aim of infection prevention and control program is to eradicate nosocomial infections but epidemiological surveillance for demonstration of performance improvement is still required to accomplish the aim. The efficient surveillance methods include data collection from multiple sources of information by trained data collectors; information should include administrative data, demographic risk factors, patients' history, diagnostic tests, and validation of data. Following the data extraction, analysis of the collected information should be done which includes description of determinants, distribution of infections, and comparison of incidence rates. Feedback and reports after analysis should be disseminated by infection control committees, management, and laboratories keeping the confidentiality of individuals. The evaluation of credibility of surveillance systems is required for effective implementations of interventions and its continuity. Finally the 
undertaking of data at regular intervals for maintenance of efficiency of surveillance systems should be made compulsory

\section{Types of Surveillance:}

a. Active Surveillance

b. Process and outcome Surveillance

c. Clinical Patient Based Surveillance

d. Lab based surveillance

e. Comprehensive surveillance

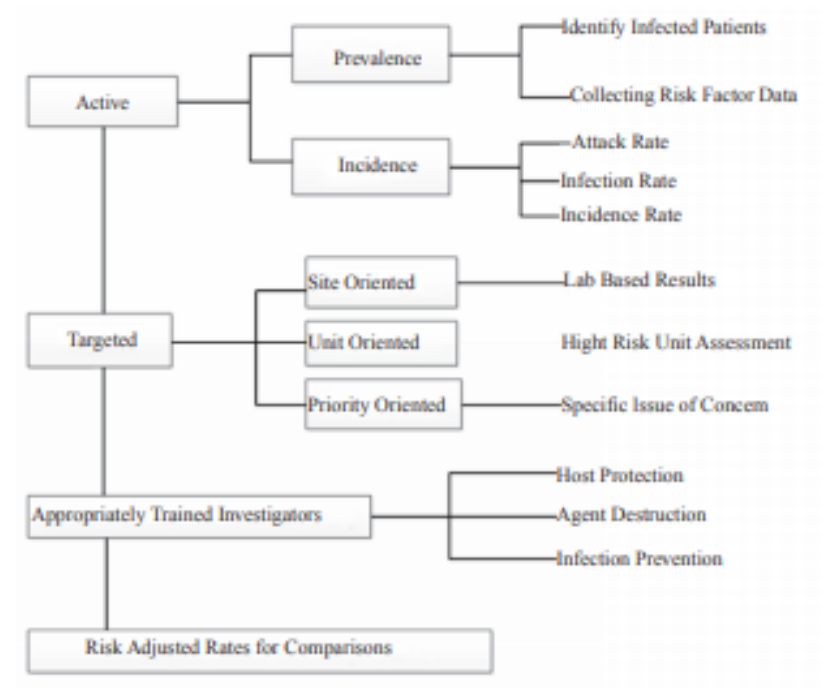

Fig 9: Organization of Surveillance (35)

\section{Management of HAI Outbreak:}

An outbreak is defined as an occurrence of disease at a rate greater than that expected within a specific geographical area over a defined time period.(34) In the context of HAIs, the geographical area may be a hospital, ward, intensive care units (ICU) or operation rooms. When there are more cases of infection with the same organism than would normally be expected in one area or period, this constitutes an outbreak. It is important to investigate a HAI outbreak immediately, as the availability and quality of microbiological evidence and epidemiological data diminishes rapidly with time between illness and investigation.

Common Pathogen Causing HAI outbreak 
$x$ Methicillin-resistant staphylococcus aureus (MRSA)

$x$ Carbapenem resistant Enterobacteriaceae (CRE)

$x$ Multi-resistant Klebsiella sp., or Pseudomonas sp.

x Diarrhoeal pathogens (e.g. Salmonella sp., Shigella sp., Campylobacter sp., norovirus)

$x$ Respiratory pathogens (e.g. infl uenza, RSV) There are also reports of HAI outbreaks caused due to M. tuberculosis, HBV, HCV, HIV, HAV, HEV, etc.

Steps of Management:

- Identification of Outbreak

- Verification of outbreak

- Confirmation of Outbreak/ Lab confirmation

- Investigation and Case construction

- Case number and Data collection

- Treatment and Follow up

- Outbreak Hypothesis

- Collect and test environmental samples

- Implement Control and preventive measures

- Follow up and Communicate findings

\section{Discussion:}

Nosocomial Infection is great threat to all the hospitals. Lots of patients affect in this disease every day. In developing countries or undeveloped countries, the rate is higher. This increase the stay inside the hospital, increase the economic burden and increase the mortality. Every country has their own guidelines, hospitals have their guidelines, WHO have their guidelines. Following the guidelines strictly, its possible to reduce the infections but a tropical country like India its very difficult to eradicate because of high temperature and humidity.

Candida is the most dangerous and Hospitals should be cautious about this. If a case detected of Candida then full hospital should be closed. 
Every hospital should have a team to monitor the rules, all the staffs should get trainings and monitoring is important. So a team of public health personal, Infectious disease, Microbiology personal should be their to monitor and updating of guidelines is important.

Asceptic measures are important during IV lines and inserting catheter. IV line and catheter should be changed regularly. NG tube, ET tube all should be changed and asceptic measures should be used. ICU should not be contaminated. A good sterilization and ventilation measures should be taken.

Self Hygiene should be maintained, Scrubs dress and other preventive measures should be taken. If any out break occurs then a strict and quick actions is necessary. It's a high time to think about antibiotic resistance. Many HAI like Klebsiella and Pseudomonas affected patients die in antibiotic resistance. A strong measures should be taken on unnecessary using of antibiotics and many genetic engineering is going in to reduce antibiotic resistance.

This Review gave a broad aspect of Nosocomial infection and Enlighted on Epidemiology, Control, Prevention Surveillance and Antibiotic Resistance

\section{Conclusion:}

With increased burden of nosocomial infections and antimicrobial resistance, it has become difficult for healthcare administrations and infection control committees to reach the goal for elimination of intervals. However, by practicing sound and healthy ways for care delivery designed by infection control committees, controlling transmission of these infections using appropriate methods for antimicrobial use, the resistance in emerging pathogens against antimicrobials can be reduced easily. An efficient surveillance method guided by WHO can help healthcare institutes to devise infection control programs. Proper training of hospital staff for biosafety, proper waste management and healthcare reforms and making general public aware of these endemic infections can also help in reduction of nosocomial infections. 
Acknowledgement: For this project I am really grateful to my Professors of Nursing in Jinan University, Guangzhou, P.R China. Their Excellent teaching and Guidance helped me to write this Review Paper.

Method: The Review is done using Pubmed, Google Scholar, Science-direct. It's a last 5 years detail review on Nosocomial infection and its management.

Work Type: Review

Funding: No Funding is present for this Work

Project: Final Exam of Fundamentals of Nursing

Conflict of Interest: Author doesn't have any COI

\section{References:}

1. Ducel G et al. Guide pratique pour la lutte contre l'infection hospitalière. WHO/BAC/79.1

2. Benenson AS. Control of communicable diseases manual, 16th edition. Washington, American Public Health Association, 1995.

3. Garner JS et al. CDC definitions for nosocomial infections, 1988. Am J Infect Control, 1988, 16:128-140.

4. Horan TC et al. CDC definitions of nosocomial surgical site infections, 1992: a modification of CDC definition of surgical wound infections. Am J Infect Control, 1992, 13:606-608.

5. McGeer A et al. Definitions of infection for surveillance in long-term care facilities. Am J Infect Control, 1991, 19:1-7.

6. WHO. The burden of health care-associated infection worldwide.2016 [Online] Available

from:http://www.who.int/gpsc/country_work/burden_hcai/en/[Accessed on 10th August, 2016]

7. DC. Types of healthcare-associated infections. Healthcare-associated infections (HAIs). 2016 [Online] Available 
from:https://www.cdc.gov/HAI/infectionTypes.html[Accessed on 10thAugust, 2016]

8. Raja Danasekaran GM, Annadurai K. Prevention of healthcareassociated infections: protecting patients, saving lives. Int J Community Med Public Health 2014; 1(1): 67-8

9. Vincent JL, Marshall J, Silva E, Anzueto A, Martin CD, Moreno R, et al. International study of the prevalence and outcomes of infection in intensive care units. JAMA 2009; 302(21): 2323-9.

10. Allegranzi B. Report on the burden of endemic health careassociated infection worldwide. Geneva: WHO; 2011.

11. Belkin, Ana et al. "Candida auris Infection Leading to Nosocomial Transmission, Israel, 2017." Emerging infectious diseases vol. 24,4 (2018): 801-804. doi:10.3201/eid2404.171715

12. Karkhane, Maryam et al. "Annual Antibiotic Related Economic Burden of Healthcare Associated Infections; a Cross-Sectional Population Based Study." Iranian journal of pharmaceutical research : IJPR vol. 15,2 (2016): 605-10.

13. Vital signs: Central line-associated blood stream infections - United States, 2001, 2008, and 2009. Morb Mortal Wkly Rep 2011; 60(08): 243-8

14. WHO. Preventing bloodstream infections from central line venous catheters. Geneva: WHO; 2016. [Online] Available from: http:// www.who.int/patientsafety/implementation/bsi/en/ [Accessed on 10th August, 2016]

15. CDC. Bloodstream infection event (central line-associated bloodstream infection and non-central line-associated bloodstream infection). Atlanta, Georgia: CDC; 2015. [Online] Available from: http://www.cdc.gov/nhsn/pdfs/pscmanual/4psc_clabscurrent.pdf [Accessed on 10th August, 2016]

16. CDC. Urinary tract infection (catheter-associated urinary tract infection [CAUTI] and non-catheter associated urinary tract infection [UTI]) and other urinary system infection [USI]) events. Atlanta, Georgia: CDC; 2016. [Online] Available from: http:// 
www.cdc.gov/nhsn/pdfs/pscmanual/7psccauticurrent.pdf [Accessed on 10th August, 2016]

17. Anderson DJ. Surgical site infections. Infect Dis Clin North Am 2011; 25(1): 135-53.

18. Owens CD. Surgical site infections: epidemiology, microbiology and prevention. J Hosp Infect 2008; 70(Suppl 2): 3-10.

19. Hunter JD. Ventilator associated pneumonia. BMJ 2012; 344: 40-4.

20. Steven M, Koenig JDT. Ventilator-associated pneumonia: diagnosis, treatment, and prevention. Clin Microbiol Rev 2006; 19(4): 637-57.

21. Moti tolera et al. Bacterial Nosocomial Infections and Antimicrobial Susceptibility Pattern among Patients Admitted at Hiwot Fana Specialized University Hospital, Eastern Ethiopia, Hindawi Advances in Medicine Volume 2018, Article ID 2127814, 7 pages https://doi.org/10.1155/2018/2127814

22. National Centre for Disease Control, Directorate General of Health Services Ministry of Health and Family Welfare, Government of India January 2020

23. CDC. HAI data and statistics. Healthcare-associated infections. Atlanta, Georgia: CDC; 2016. [Online] Available from: http:// www.cdc.gov/HAI/surveillance/index.html [Accessed on 10th August, 2016]

24. Nosocomial infections: Epidemiology, prevention, control and surveillance, Asian Pac J Trop Biomed (2017), http://dx.doi.org/10.1016/j.apjtb.2017.01.019

25. Pradhan NP, Bhat SM, and Ghadage DP. Nosocomial infections in the medical ICU: a retrospective study highlighting their prevalence, microbiological profi le and impact on ICU stay and mortality. $J$ Assoc Physicians India. 2014;62:18-21

26. Klompas M, Branson R, Eichenwald EC, Greene LR, Howell MD, Lee Get al. Strategies to prevent ventilator-associated pneumonia in acute care hospitals: 2014 update. Infect Control Hosp Epidemiol. 2014;35:915-36.

27. Hooton TM, Bradley SF, Cardenas DD, Colgan R, Geerlings SE, Rice JC et al. Diagnosis, prevention, and treatment of catheter-associated urinary tract infection in adults: 2009 International Clinical Practice Guidelines from the Infectious Diseases Society of America. Clin Infect Dis. 2010;50:625-63. 
28. Ducel, Georges, Jacques Fabry, and L. Nicolle. "Prevention of hospital acquired infections: a practical guide." Prevention of hospital acquired infections: a practical guide. Ed. 2 (2002).

29. ] Colgan R. Appropriate antimicrobial prescribing: approaches that limit antibiotic resistance. Am Fam Physician 2001; 64(6): 999- 1005.

30. Singh PK. Antibiotics, handle with care. Geneva: WHO; 2016. [Online] Available from: http://www.searo.who.int/mediacentre/ releases/2015/antibiotics-awareness-week-2015/en/ [Accessed on 10th August, 2016]

31. WHO. Antimicrobial resistance. Geneva: WHO; 2014. [Online] Available from: http://www.searo.who.int/thailand/factsheets/fs0023/en/ [Accessed on 10th August, 2016]

32. WHO. WHO's first global report on antibiotic resistance reveals serious, worldwide threat to public health. Geneva: WHO; 2014.

33. WHO. Urgent action needed to prevent a return to pre-antibiotic era: WHO. Geneva: WHO; 2015. [Online] Available from: http://www.searo.who.int/mediacentre/releases/2015/1612/en/[Accessed on 10th August, 2016]

34. Beck-Sague C, Jarvis WR, Martone WJ. Outbreak investigations. Infect Control Hosp Epidemiol. 1997;18:138-45 (http://www.ncbi.nlm.nih.gov/pubmed/9120245, accessed 15 October 2019)

35. Aitken CJD. Nosocomial spread of viral disease. Clin Microbiol Rev 2001; 14(3): 528-46. 